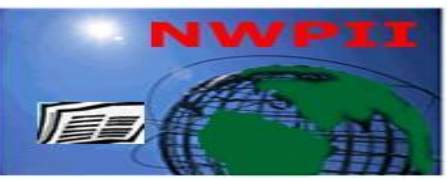

American Journal of Biomedical Sciences

ISSN: 1937-9080

nwpii.com/ajbms

\title{
Image Fusion Using Contourlet for Enhancement and Wavelet Transform: to Aid Medical Diagnosis
}

\author{
A. Bengana*, M.A.Chikh, I. Boukli hacene
}

Biomedical Laboratory, Department of Electrics and Electronics, Technology Faculty, University Of Tlemcen 13000, Algeria.

*Corresponding Author

A. Bengana

Department of electrics and Electronics

Technology Faculty

University Of Tlemcen 13000

Algeria

Email: befatih80@yahoo.fr

Received: 26 March 2016; | Revised: 7 June 2016; | Accepted: 16 July 2016

\begin{abstract}
Medical image fusion is the process of combining multiple images from single or multimodal medical image. In this article, we have presented an hybrid image fusion based on non-subsampled contourlet (NSCT) enhancement to highlight the presentation of the original image appearance taking into account the difficulties of classical methods linked to the traditional enhancement methods. This algorithm is performed in CDF 9/7 wavelet transform based on lifting scheme to combine magnetic resonance imaging (MRI) and Positron emission tomography (PET) with applying fusion rules. The coefficients of the low frequency band are combined by a maximal energy rule and coefficients of the high frequency bands are combined by a maximal variance rule. Finally, the results of image fusion are evaluated with mutual information (MI) and spatial frequency (SF) then compared with the existent methods such as (PCA, DWT).
\end{abstract}

Keywords: Medical image, Image fusion, NSCT, Lifting scheme, Wavelet CDF9/7.

\section{Introduction}

Image fusion in medical has become the focus of research now. Image fusion based on multi-resolution method can be divided into three types [1]: the first type is based on the pyramid decomposition.

The second type is based on wavelet decomposition, such as discrete wavelet transform [2], wavelet packet [3], multi-wavelet transform [4-5-6]. The third type is the secondgeneration wavelet transform (SGWT), such as image fusion based on curvelet transform [7], Nonsubsampled contourlet transform [8], and a medical image fusion algorithm based on lifting wavelet transform [9]. In [10] are proposed the performance analysis of multi source fused medical images using multiresolution transforms. In [11] are developed a new algorithm of multimodal medical image fusion using modified 
daubechies wavelet transform. In the recent research [12], we cited the new approach of data fusion for cardiac resynchronization therapy optimization.

We introduce in this paper a hybrid image fusion algorithm based in modified (NSCT) nonsubsampled contourlet coefficients of images in corresponding subbands via a new and operable nonlinear mapping function [13-24] and take the noise into account for more precise reconstruction and better visualization. The second step for our algorithm we applied the wavelet transform CDF 9/7 based on lifting scheme [14]. The algorithm may consider both of the result and speed of the fusion with tools of wavelet based on lifting scheme. In addition, we compare evaluation parameters results obtained with the existing techniques namely the PCA and DWT.
The organization of this paper is as follows, the Section 2 explains the non-subsampled contourlet transform for Contrast-enhancement and In Section 3 biorthogonal wavelet CDF 9/7 and proposed method are presented. In Section 4 we present the evaluations parameters. In Section 5. The experimental results are shows and we compared with the existing techniques. Finally the conclusion in Section 6.

\section{Non Subsampled Contourlet Transform}

Cunha and al [17] propose the NSCT (figure 1), which is a shift-invariant version of the contourlet transform. NSCT not only has the multiscale and time-frequency-localization properties of wavelets, but also offers a high degree of directionality, anisotropy and shiftinvariance, thus, can effectively capture geometry and directional information of images.

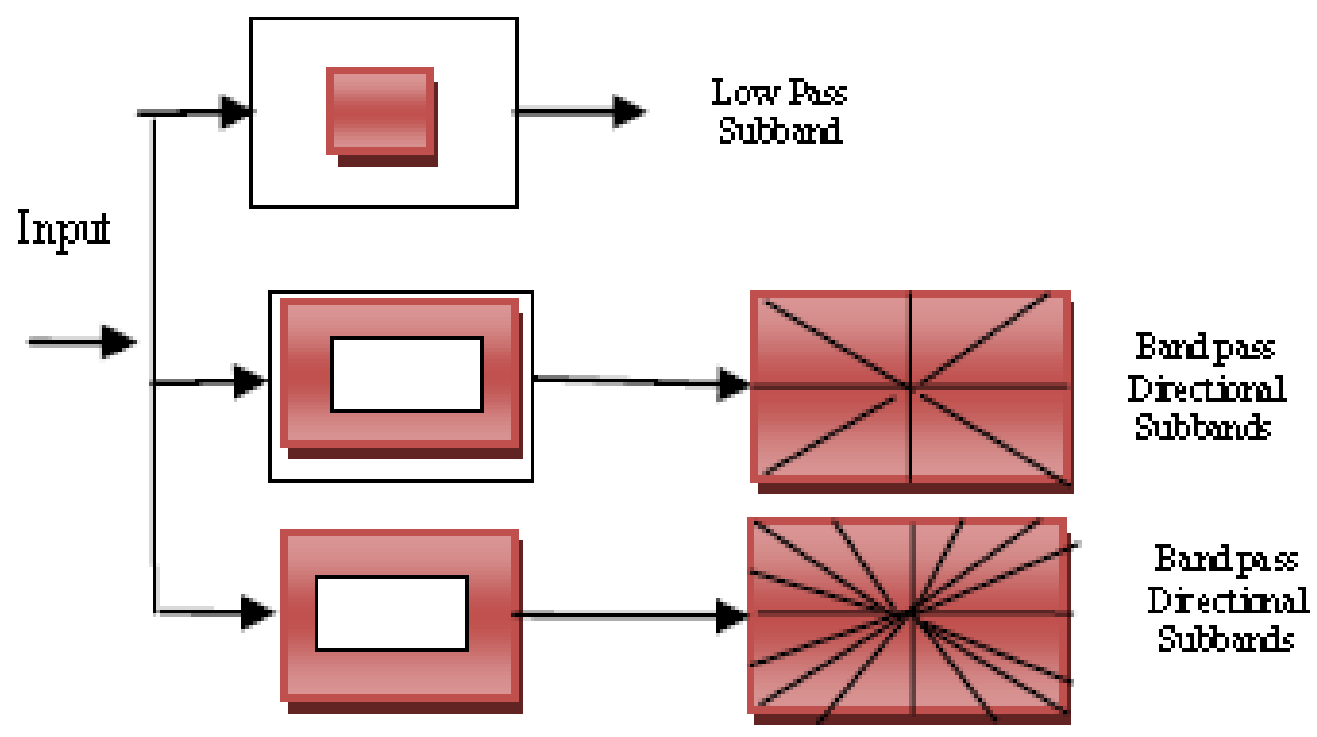

Figure 1. The NSCT decomposition.

\section{CDF9/7 based on lifting scheme [18]}

These wavelets are part of the family of symmetric biorthogonal wavelet CDF. The low pass filters associated with wavelet $9 / 7$ have $p=9$ coefficients in the analysis, $p=7$ coefficients to synthesize.
The wavelets $9 / 7$ have a great number of null moments for a relatively short support. They are more symmetrical and very close to orthogonality. Antonini and al [19] were the first to show the superiority of the biorthogonal wavelet transform 9/7 for the decorrelation of natural images and is used by the JPEG-2000 [20-23]. 
The Lifting scheme of the biorthogonal transform 9/7 goes through of four steps: two prediction operators and two update operators as shown it figure 2 [21-22]. The synthesis side of the filter bank simply inverts the scaling, and reverses the sequence of the lifting and update steps.

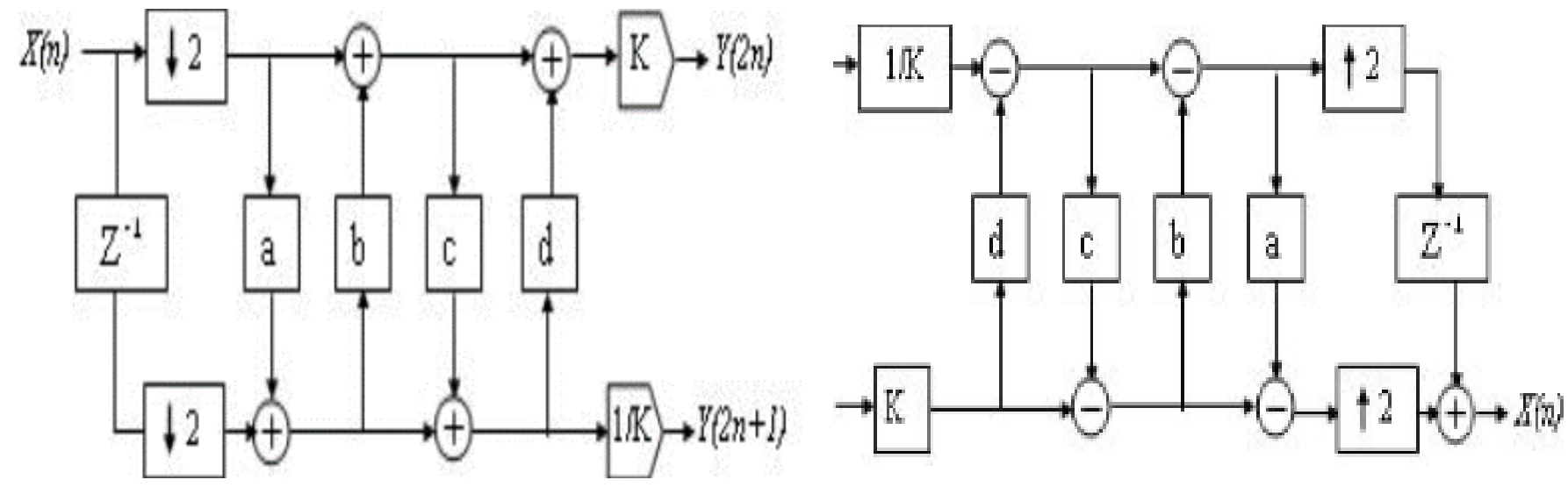

Figure 2. Lifting implementation of the Analysis / Synthesis side of the CDF 9/7 wavelet.

Finally, our proposed method can be summarized in the following steps (is the continuation of the article[15]).

Step 1: Contrast-enhancement using NSCT :

- Applied NSCT for each input images using five scales of decomposition and 4 , $8,8,16,16$ directions in the scales from coarser to finer respectively and obtain the NSCT coefficients

- Estimate the noise standard deviation with the median operator [16]; and average energy distribution of standard white noise in each subband

- Modify the NSCT coefficients for each subband via the proposed nonlinear mapping function in [13]

- Reconstruct the enhanced image from the modified coefficients for two image

Step 2: Decompose the enhanced image using three level by wavelet transform based lifting scheme LWT (Biorthogonal CDF 9/7) apply our algorithm on each image independently.

Step 3: Applied the fusion rules according to lower subband equation (1) and higher subband equation (2) defined in section 2.

Step 4: Reconstruct image fused by taking inverse LWT (CDF 9/7).

\section{- Rules for lower subband coefficient}

The approximation coefficients is a smooth and sub sampled version of the original image. Therefore, most of the source images information is kept in low frequency bands. The proposed selection principles for the sub band coefficients are finally defined as the maximum selection rule is:

$$
\begin{aligned}
& L L_{F}=\left\{\begin{array}{lll}
L L_{A} & \text { if } & L L_{A}>L L_{B} \\
L L_{B} & \text { if } & L L_{A} \leq L L_{B}
\end{array}\right. \\
& \text { - Rules for higher subband coefficient }
\end{aligned}
$$

The high pass sub band coefficients represent the detailed components of the source image. Therefore, we propose a scheme by computing the variance rule method to select the high frequency coefficients.

$L H_{F}=\left\{\begin{array}{ccc}L H_{A} & \text { if } & \operatorname{Var}_{L H_{A}}>\operatorname{Var}_{L H_{B}} \\ L H_{B} & \text { if } & \operatorname{Var}_{L H_{A}}<\operatorname{Var}_{L H_{B}} \\ \frac{\left(L H_{A}+L H_{B}\right)}{2} & \text { otherwise }\end{array}\right.$

Apply the same technique for: $H L_{A}, H H_{A}, H L_{B}, H H_{B}$. 


\section{Evaluations parameters}

In the present work, we have used two performance measures to evaluate the performance of hybrid fusion algorithm (NSCT+CDF9/7 LWT).

\subsection{Mutual Information (MI)}

Compares the image source and the fused image, more value is small, the relationship between the two images is non-existent.

$$
\mathrm{MI}=\quad \sum_{\mathrm{af}} \mathrm{P}_{\mathrm{AF}}(\mathrm{a}, \mathrm{f}) \log \frac{\mathrm{P}_{\mathrm{AF}}(\mathrm{a}, \mathrm{f})}{\mathrm{P}_{\mathrm{A}}(\mathrm{a}) \mathrm{P}_{\mathrm{F}}(\mathrm{f})}
$$

$\mathrm{P}_{\mathrm{AF}}(\mathrm{a}, \mathrm{f})$ The joint histogram between fused image $\mathrm{F}$ and the source image $\mathrm{A}$.

\subsection{Spatial Frequency (SF)}

It measures the total activity and the level of clarity of an image, an important value mean that the result of fusion is good.

$$
\mathrm{SF}=\sqrt{\mathrm{RF}^{2}+\mathrm{CF}^{2}}
$$

Where RF and CF are the row frequency and the column frequency respectively.

\section{Results and Discussion}

In the first, we used the NSCT toolbox; while for the contourlet transform, we choose the biorthogonal Daubechies "9-7" filters for the laplacian stage and the fan filters. It's clear that the medical image contrast has been improved by proposed NSCT-based enhancement approach In [17] with $p=0.75$ where $P$ is a constant which controls the shape of the mapping function as well as the enhancement rate.

We applied our algorithm (NSCT+ CDF9/7 LWT), on different multisource images MRI, CT $\&$ PET are downloaded from (www.imagefusion.org) of size $(512 \times 512)$, encoded by $8 \mathrm{bpp}$, we calculated the parameters for assessing quality (SF, MI) to extend the effectiveness of this algorithm. These images were tested on Intel Core (I3) $2.13 \mathrm{GHz}$ PC with 2GB of RAM using Matlab 2010a.

To show the performance of our method, we will now make a comparison between these different types of transform Principal Component Analysis (PCA) and Discrete Wavelet Transform (DWT). The fused image output based on proposed methods illustrated in Figure 3, 4 and 5, then the performances of fused medical images are shown in Figure 6.

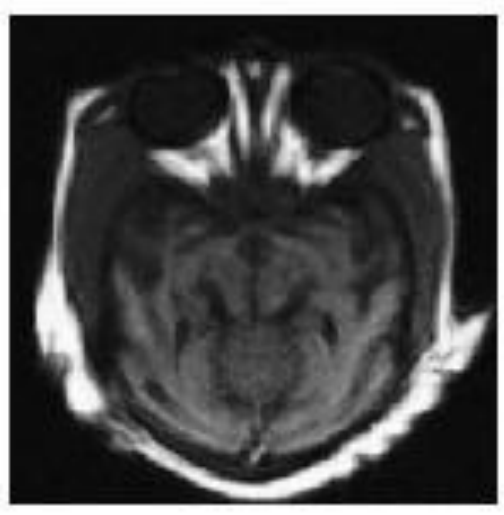

(a)

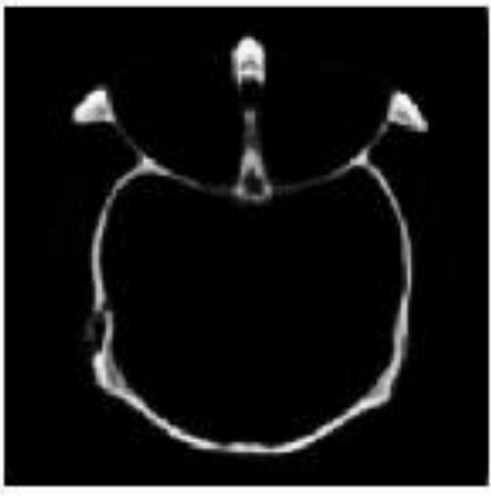

(b)

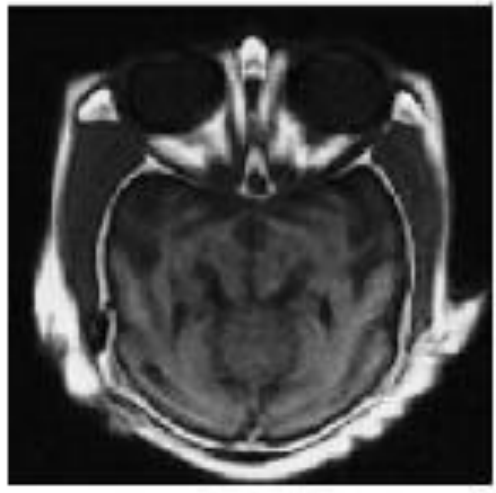

(c)

Figure 3. MRI fusion results, (a) image (MRI), (b) image (CT), (c) Fused image (NSCT+CDF97LWT) 


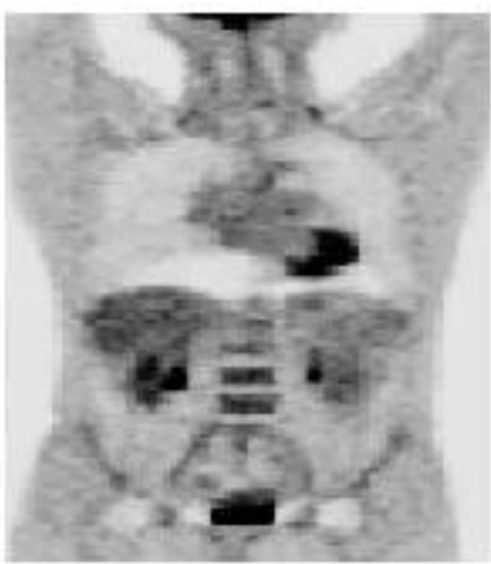

(a)

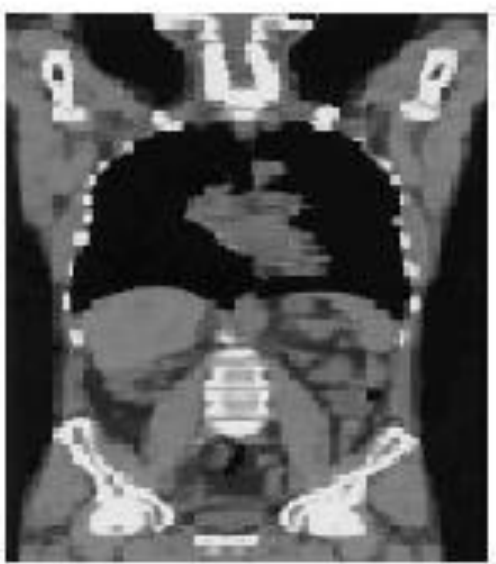

(b)

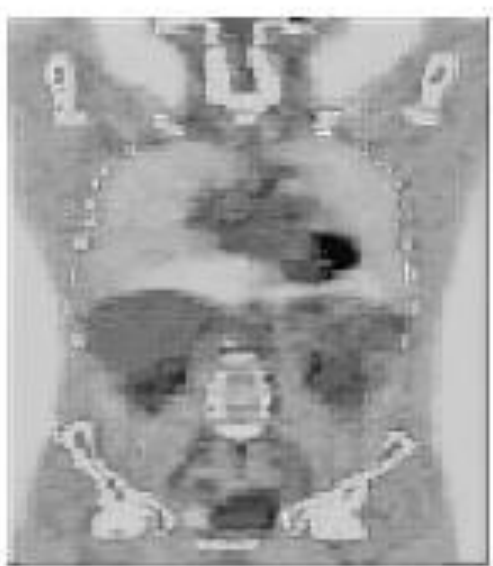

(c)

Figure 4. CT-PET fusion results (a) image (PET), (b) image (CT), (c) Fused image (NSCT+CDF97LWT)

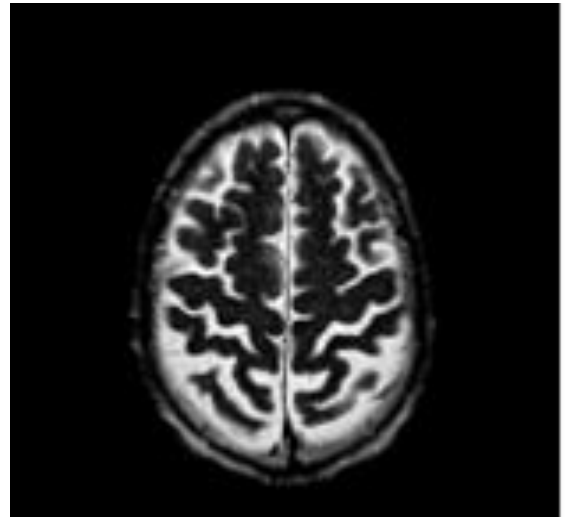

(a)

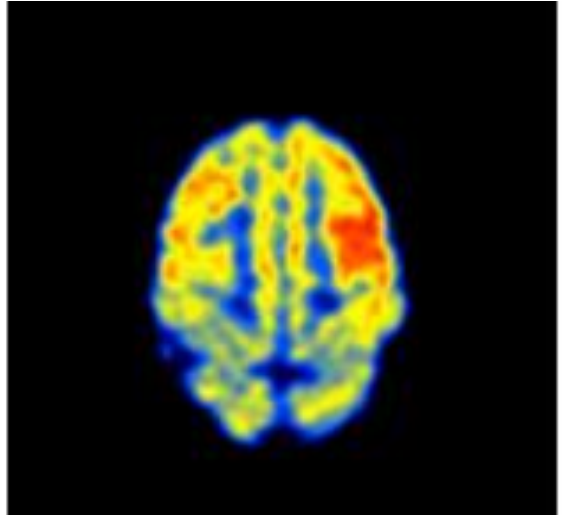

(b)

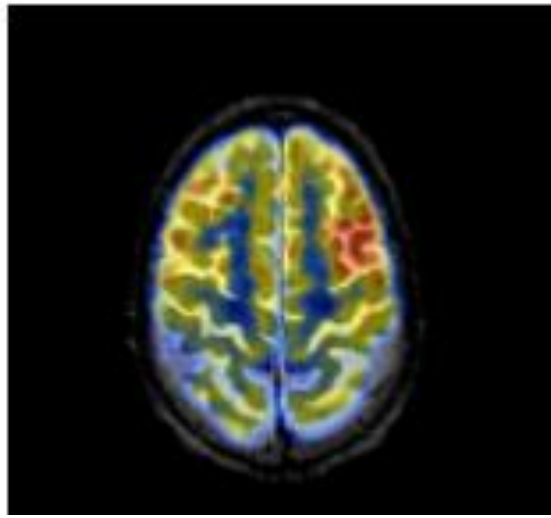

(c)

Figure 5. MRI-PET fusion results (a) image (MRI), (b) image (PET), (c) Fused image (NSCT+CDF97LWT)
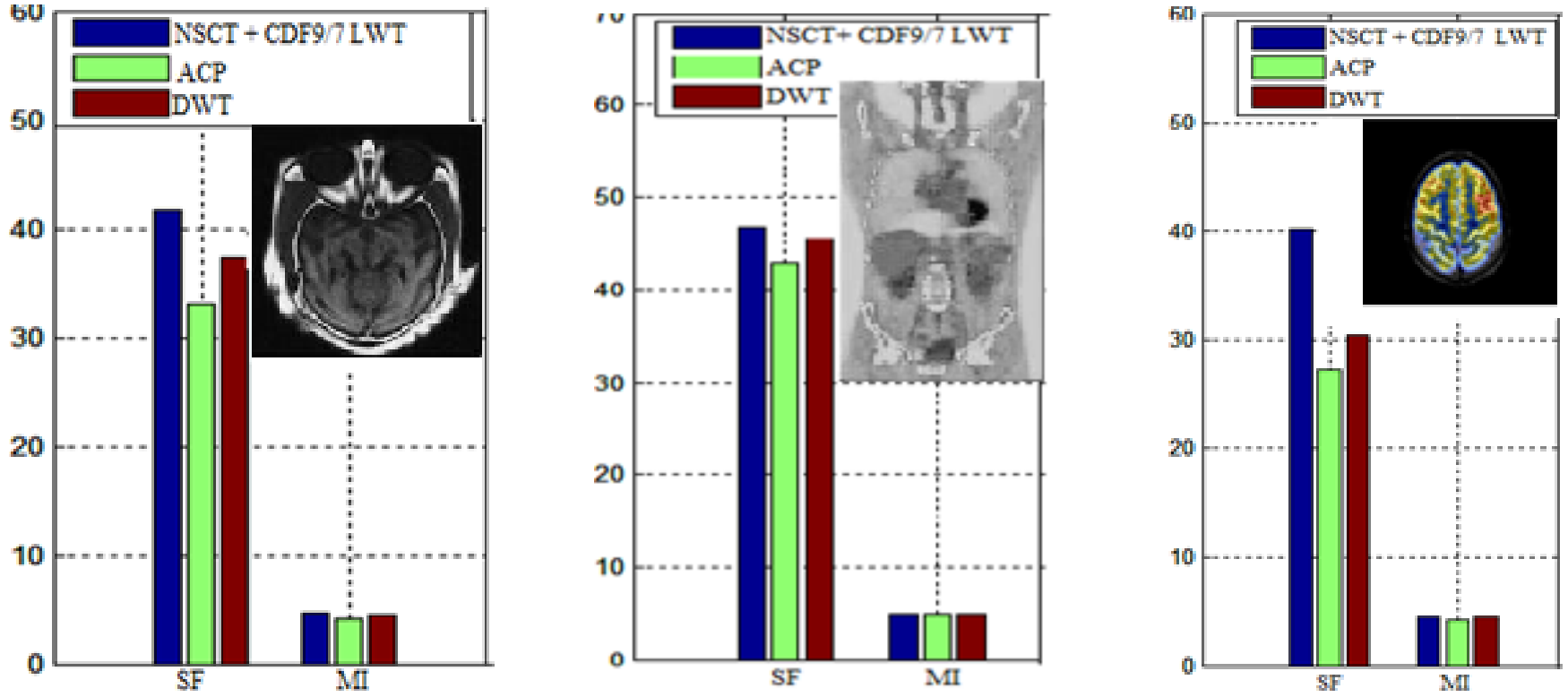

Figure 6. Variations of evaluation parameters with different fusion methods. 


\section{Conclusion}

In this paper, the essential objective is the diagnosis aid of medical images after the multisource images fusion. We used the CDF9/7 based on lifting scheme with NSCT toolbox enhancement to obtain fused image. After, several applications were compared with (PCA and DWT). These results are Performed and analyzed with calculate different evaluation parameters. Finally, we found the proposed algorithm that gives better results than the other fusion methods.

In perspective, we try to extend our study to annotated databases. We aspire to apply our algorithm to fusion 3D medical images and video sequences.

\section{References:}

[1] Hu, G., Liu Z and Xu X. (2008). 'Research and Recent Revelopment of ImageFusion at Pixel Level'. Application Research of Computers, Vol.25, No.3, (March 2008), pp. 650-655.

[2] Li, .M and Wu S, J. (2003).' A New Image Fusion Algorithm Based on Wavelet Transform', Proceedings of International Conference on Computational Intelligence and Multimedia Applications, pp. 154-159, Phoenix, Arizona, USA, May 17-22, 2003.

[3] Chunlin Zhao and all (2010). 'Physiological Assessment of Driving Mental Fatigue Using Wavelet Packet Energy and Random Forests' .Am. J. Biomed. Sci. 2010, 2(3), 262-274; doi: 10.5099/aj100300262.

[4] Wang H. (2004) 'A New Multiwaveletbased Approach to Image Fusion'. Journal of Mathematical Imaging and Vision, Vol.21, No.3, (March 2004), pp. 177-192.

[5] Zhang X, Pan Q and Zhao Y.(2005). 'Image Fusion Method Based on Stationary Multiwavelet Transform'. Journal of Optoelectronics Laser, Vol.16, No.5, (May 2005), pp. 605-609.

[6] Wang Z, Yu X and Zhang L.B.(2008). 'A Remote Sensing Image Fusion Algorithm Based on Integer Wavelet Transform'.
Journal of Optoelectronics Laser, Vol.19, No.11, (November 2008), pp. 1542-1545.

[7] Filippo N, Andrea G and Stefano B.(2007)

'Remote Sensing Image Fusion Using the Curvelet Transform'. Information Fusion, Vol.8, 2007, pp. 143-156.

[8] Yang X.H. and Jiao L.C. (2008)'Fusion Algorithm for Remote Sensing Images Based on Nonsubsampled Contourlet Transform'. Acta Automatica Sinica, Vol.34, No.3, (March 2008), pp. 274-281.

[9] Xue-jun, W., and M. Ying, (2010) 'A medical image fusion algorithm based on lifting wavelet transform', in: Artificial Intelligence and Computational Intelligence (AICI), 2010 International Conference on, Vol. 3, IEEE, 2010, pp. 474-476.

[10] Hima Bindu, Ch., K.Satya Prasad,.(2012) 'Performance Analysis Of Multi Source Fused Medical Images Using Multiresolution Transforms', International Journal of Advanced Computer Science and Applications, Vol. 3, No. 10, 2012.

[11] Kanisetty Venkata Swathi and Hima Bindu CH. (2013) 'Modified Approach of Multimodal Medical Image Fusion Using Daubechies Wavelet Transform' International Journal of Advanced Research in Computer and Communication Engineering Vol. 2, Issue 11, November 2013.

[12] François .T, Antoine .S, Christophe .L, Erwan. D, Alfredo. I, Mireille.G, (2014) 'Multimodal Registration and Data Fusion for Cardiac Resynchronization Therapy Optimization' IEEE Trans. On Medical Imaging, (March 2014) 0278-0062.

[13] Kang Li, Xuejun Chen, Xiangjiang $\mathrm{Hu}$, Xiang Shi, Long Zhang "Image Denoising and Contrast Enhancement Based on Nonsubsampled Contourlet Transform" IEEE Trans. Image Process, vol. 19, no. 3, pp. 131-135, 2010.

[14] Beladgham M., A. Bessaid, A. Moulay Lakhdar and A. Taleb Ahmed, (2011). 'Improving quality of medical image compression using biorthogonal $\mathrm{CDF}$ wavelet based on lifting scheme and SPIHT 
coding', Serbian Journal of Electrical Engineering, Vol 8, Issue 2, ISSN 14514869, pp 163-179, 이: 10.2298/SJEE1102163B, 2011.

[15] Bengana .A, Boukli H. I, Chikh. M.A. (2015). 'MRI-T1 and T2 Image Fusion for Brain image using CDF wavelet based on Lifting Scheme', Global Journal Of Medical Research, Vol 15,Issue 6, ISSN 2249-4618, pp 28-33, 2015.

[16] Chang S.G, B. Yu, M.Vetterli (2000). "Spatially adaptive wavelet thresholding with context modeling for image denoising". IEEE Trans. on Image Processing, Vol. 9, No. 9, pp. 1522-1531.

[17] Cunha A. L, J. Zhou, and M. N. Do, "The non subsampled contourlet transform: Theory, design, and applications," IEEE Trans. Image Process, vol. 15, no. 10, pp. 3089-3101, Oct. 2006.

[18] Boukli H.I, Bessaid. A. (2014). 'Hybrid colour medical image compression by $\mathrm{CDF}$ wavelet and cosine transforms' Int. J. Biomedical Engineering and Technology, Vol. 16, No. 1, 2014.

[19] Antonini M., Barlaud, M., Mathieu, P. and Daubechies, I. (1992). 'Image coding using wavelet transform'. IEEE Trans. Image Processing, Vol. 1, April 1992, pp.205-220.
[20] Manuela, F., Dimitri, V.D. and Michael, U. (2005) 'An orthogonal family of quincunx wavelets with continuously adjustable order', IEEE Trans. Image Processing, Vol. 14, No. 4.

[21] Sweldens, W. (1996). 'The lifting scheme: A custom-design construction of biorthogonal wavelets', Appl Comput Harmon Anal, pp. 186-200, 1996.

[22] Calderbank, A.R., I. Daubechies, W. Sweldens, and BL. Yeo, (1998). 'Wavelet transforms that map integers to integers', Appl Comput Harmon Anal, 5, pp. 332-369, 1998.

[23] Duong Tr Luo and all (2016). 'Advanced Two-State Compressing Algorithm: A Versatile, Reliable and Low-Cost Computational Method for ECG Wireless Applications', Am. J. Biomed. Sci. 2016, 8(1), 1-23; doi: 10.5099/aj160100001.

[24] Frederick Valeriote, Ben Chen, Joseph Media, Kevin R. Bobbitt, Jiajiu Shaw (2015). 'Enhancement of Bone Marrow CD41+ Megakaryocytes as well as Modulation of TNF-a, IL-12, and IL-5 by a Novel Small Molecule, UTL-5g, in Mice Treated with Cisplatin', Am. J. Biomed. Sci. 2015, 7(1), 1-8; doi: 10.5099/aj150100001. 